Dept. of Math. University of Oslo

\title{
Optimal Control of Stochastic Partial Differential Equations
}

\author{
Bernt Øksendal \\ Center of Mathematics for Applications (CMA), \\ Dept. of Mathematics, University of Oslo \\ Box 1053 Blindern, N-0316 Oslo, Norway \\ (email: oksendal@math.uio.no) \\ and \\ Norwegian School of Economics and Business Administration, \\ Helleveien 30, N-5045 Bergen, Norway \\ Revised March 15, 2005

\begin{abstract}
We prove a sufficient maximum principle for the optimal control of systems described by a quasilinear stochastic heat equation. The result is applied to solve a problem of optimal harvesting from a system described by a stochastic reaction-diffusion equation.
\end{abstract}

Key words: Optimal control, stochastic forward and backward partial differential equations, stochastic maximum principle.

MSC 2000: Primary 93E20, Secondary 60H15, 60G35, 93E11, 62M20.

\section{Introduction}

Let $T>0$ and let $G$ be an open set in $\mathbb{R}^{n}$ with $C^{1}$ boundary $\partial G$. Suppose that the state $Y(t, x) \in \mathbb{R}$ of a system at time $t \in[0, T]$ and at the point $x \in \bar{G}=G \cup \partial G$ is given by a quasilinear stochastic heat equation of the form

$$
\begin{aligned}
& d Y(t, x)=\left\{\begin{array}{r}
{[L Y(t, x)+b(t, x, Y(t, x), u(t, x))] d t} \\
+\sigma(t, x, Y(t, x), u(t, x)) d B(t) ; \quad(t, x) \in(0, T) \times G
\end{array}\right. \\
& Y(0, x)=\xi(x) ; \quad x \in \bar{G} \\
& Y(t, x)=\eta(t, x) ; \quad(t, x) \in(0, T) \times \partial G .
\end{aligned}
$$


Here $d Y(t, x)$ denotes the Itô differential with respect to $t$, while $L$ is a second order partial differential operator acting on $x$ given by

$$
L \phi(x)=\sum_{i, j=1}^{n} a_{i j}(x) \frac{\partial^{2} \phi}{\partial x_{i} \partial x_{j}}+\sum_{i=1}^{n} b_{i}(x) \frac{\partial \phi}{\partial x_{i}} ; \quad \phi \in C^{2}\left(\mathbb{R}^{n}\right)
$$

where $a(x)=\left[a_{i j}(x)\right]_{1 \leq i, j \leq n}$ is a given symmetric nonnegative definite symmetric $n \times n$ matrix with entries $a_{i j}(x) \in C^{2}(G) \cap C(\bar{G})$ for all $i, j=1,2, \ldots, n$ and $b_{i}(x) \in C^{2}(G) \cap C(\bar{G})$ for $i=1,2, \ldots, n$. The process $B(t)=B(t, \omega) ; t \geq 0, \omega \in \Omega$ is a (1-dimensional, 1-parameter) Brownian motion on a filtered probability space $\left(\Omega, \mathcal{F},\left\{\mathcal{F}_{t}\right\}_{t \geq 0}, P\right)$, while $u(t, x)=u(t, x, \omega)$ is our control process. We assume that $u(t, x)$ has values in a given convex set $U \subset \mathbb{R}^{k}$ and that $u(t, x, \cdot)$ is $\mathcal{F}_{t}$-measurable for all $(t, x) \in(0, T) \times G$ i.e. that $u(t, x)$ is adapted for all $x \in G$. The functions $b:[0, T] \times G \times \mathbb{R} \times U \rightarrow \mathbb{R}$ and $\sigma:[0, T] \times G \times \mathbb{R} \times U \rightarrow \mathbb{R}$ are given $C^{1}$ functions. The boundary value functions $\xi: \bar{G} \rightarrow \mathbb{R}$ and $\eta:[0, T] \times \partial G \rightarrow \mathbb{R}$ are assumed to be deterministic and $C^{1}$.

We call the control process $u(t, x)$ admissible if the corresponding stochastic partial differential equation (1.1)-(1.3) has a unique, strong solution $Y(\cdot) \in L^{2}(\lambda \times P)$, where $\lambda$ is Lebesgue measure on $[0, T] \times \bar{G}$, and with values in a given set $S \subset \mathbb{R}$. The set of admissible controls is denoted by $\mathcal{A}$.

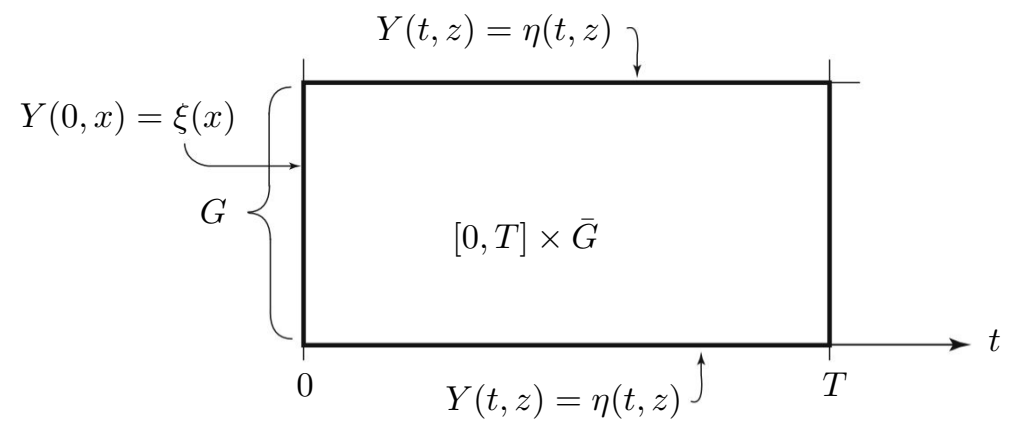

Figure 1: The boundary values of $Y(t, x)$.

Suppose the performance $J(u)$ obtained by applying the control $u \in \mathcal{A}$ has the form

$$
J(u)=E\left[\int_{0}^{T}\left(\int_{G} f(t, x, Y(t, x), u(t, x)) d x\right) d t+\int_{G} g(x, Y(T, x)) d x\right]
$$

where $f$ and $g$ are given lower bounded $C^{1}$ functions and $E$ denotes the expectation with respect to $P$.

We consider the problem to find $J^{*} \in \mathbb{R}$ and $u^{*} \in \mathcal{A}$ such that

$$
J^{*}=\sup _{u \in \mathcal{A}} J(u)=J\left(u^{*}\right)
$$

This is an optimal control problem for the quasilinear stochastic heat equation.

The main purpose of this paper is to prove a maximum principle type of verification theorems for such optimal control problems (Theorems 2.1, 2.2 and 2.3). Then we use the 
connection between such optimal control problems (with complete information) and stochastic control problems with partial observation to establish a sufficient maximum principle for partial observation control (Theorem 3.1).

Stochastic control of the stochastic partial differential equations (SPDEs) arizing from partial observation control has been studied by Mortensen [M], using a dynamic programming approach, and subsequently by Bensoussan, using a maximum principle method. See [B3] and the references therein. Our approach differs from the approach of Bensoussan in two ways: First, we give sufficient maximum principle results, not necessary ones. Second, we consider more general quasilinear semielliptic SPDEs.

Here is an outline of the paper: In Section 2 we give 3 versions of a sufficient maximum principle (verification theorem) for optimal control of quasilinear SPDEs. In Section 3 the results are illustrated by solving a problem of optimal harvesting from a system described by a stochastic reaction-diffusion equation.

\section{A Sufficient Maximum Principle}

We now formulate a sufficient maximum principle for the optimal control of the problem (1.1)-(1.6).

Define the Hamiltonian $H:[0, T] \times G \times \mathbb{R} \times U \times \mathbb{R} \times \mathbb{R} \rightarrow \mathbb{R}$ associated to the problem (1.1)-(1.6) by

$$
H(t, x, y, u, p, q)=f(t, x, y, u)+b(t, x, y, u) p+\sigma(t, x, y, u) q .
$$

Let

$$
L^{*} \phi(x)=\sum_{i, j=1}^{n} \frac{\partial^{2}}{\partial x_{i} \partial x_{j}}\left(a_{i j}(x) \phi(x)\right)-\sum_{i=1}^{n} \frac{\partial}{\partial x_{i}}\left(b_{i}(x) \phi(x)\right)
$$

be the adjoint of the operator $L$ given in (1.4). For each $u \in \mathcal{A}$ we consider the following adjoint backward SPDE in the two unknown adapted processes $p(t, x), q(t, x)$ :

$$
\begin{aligned}
& d p(t, x)=-\left\{\left(\frac{\partial H}{\partial y}\right)(t, x, Y(t, x), u(t, x), p(t, x), q(t, x))\right. \\
& \left.\quad+L^{*} p(t, x)\right\} d t+q(t, x) d B(t) ; \quad 0 \leq t \leq T, \quad x \in G \\
& \text { 4) } \quad p(T, x)=\frac{\partial g}{\partial y}(x, Y(T, x)) ; \quad x \in \bar{G} \\
& \quad p(t, x)=0 ; \quad(t, x) \in(0, T) \times \partial G
\end{aligned}
$$

Here $Y(t, x)=Y^{u}(t, x)$ is the solution of (1.1)-(1.3) corresponding to $u$.

\section{Theorem 2.1 (Sufficient SPDE maximum principle I)}

Let $\hat{u} \in \mathcal{A}$ with corresponding solution $\widehat{Y}$ of (1.1)-(1.3) and let $\hat{p}(t, x), \hat{q}(t, x)$ be a solution of the associated adjoint backward SPDE (2.3)-(2.5). Suppose the following, (2.6)-(2.9), hold: 


\section{The functions}

$(y, u) \rightarrow H(y, u):=H(t, x, y, u, \hat{p}(t, x), \hat{q}(t, x)) ; y \in \mathbb{R}, u \in U$ and

$y \rightarrow g(x, y) ; \quad y \in \mathbb{R}$ are concave, for all $(t, x) \in[0, T] \times G$

$$
H(t, x, \widehat{Y}(t, x), \hat{u}(t, x), \hat{p}(t, x), \hat{q}(t, x))=\sup _{u \in U} H(t, x, \widehat{Y}(t, x), u, \hat{p}(t, x), \hat{q}(t, x))
$$

for all $(t, x) \in[0, T] \times G$

For all $u \in \mathcal{A}$, with $Y(t, x)=Y^{(u)}(t, x)$,

$$
E\left[\int_{G} \int_{0}^{T}(Y(t, x)-\widehat{Y}(t, x))^{2} \hat{q}^{2}(t, x) d t d x\right]<\infty
$$

and

$$
E\left[\int_{G} \int_{0}^{T} \hat{p}(t)^{2} \sigma^{2}(t, x, Y(t, x), u(t, x)) d t d x\right]<\infty
$$

Then $\hat{u}(t, x)$ is an optimal control for the stochastic control problem (1.6).

Proof. Let $u$ be an arbitrary admissible control with corresponding solution $Y(t, x)=$ $Y^{u}(t, x)$ of (1.1)-(1.3). Consider

$$
J(\hat{u})-J(u)=E\left[\int_{0}^{T} \int_{G}\{\hat{f}-f\} d x d t+\int_{G}\{\hat{g}-g\} d x\right]
$$

where

$$
\begin{aligned}
\hat{f} & =f(t, x, \widehat{Y}(t, x), \hat{u}(t, x)), & & f=f(t, x, Y(t, x), u(t, x)) \\
\hat{g} & =g(x, \widehat{Y}(T, x)) \quad \text { and } & g & =g(x, Y(T, x)) .
\end{aligned}
$$

Similarly we put

$$
\begin{array}{ll}
\hat{b}=b(t, x, \widehat{Y}(t, x), \hat{u}(t, x)), & b=b(t, x, Y(t, x), u(t, x)) \\
\hat{\sigma}=\sigma(t, x, \widehat{Y}(t, x), \hat{u}(t, x)), & \sigma=\sigma(t, x, Y(t, x), u(t, x))
\end{array}
$$

and we set

$$
\begin{aligned}
\widehat{H} & =H(t, x, \widehat{Y}(t, x), \hat{u}(t, x), \hat{p}(t, x), \hat{q}(t, x)), \\
H & =H(t, x, Y(t, x), u(t, x), \hat{p}(t, x), \hat{q}(t, x)) .
\end{aligned}
$$

Then (2.10) can be written

$$
\begin{gathered}
J(\hat{u})-J(u)=I_{1}+I_{2}, \quad \text { where } \\
I_{1}=E\left[\int_{0}^{T} \int_{G}\{\widehat{H}-H-(\hat{b}-b) \hat{p}-(\hat{\sigma}-\sigma) \hat{q}\} d x d t\right]
\end{gathered}
$$

and

$$
I_{2}=E\left[\int_{G}\{\hat{g}-g\} d x\right] .
$$


By concavity of the function $y \rightarrow g(x, y)$ we have

$$
g-\hat{g} \leq \frac{\partial g}{\partial y}(x, \widehat{Y}(T, x)) \cdot(Y(T, x)-\widehat{Y}(T, x))
$$

Therefore, writing

$$
\widetilde{Y}(t, x):=Y(t, x)-\widehat{Y}(t, x),
$$

we get

$$
\begin{aligned}
I_{2} \geq-E & {\left[\int_{G} \frac{\partial g}{\partial y}(x, \widehat{Y}(T, x)) \cdot \tilde{Y}(T, x) d x\right] } \\
=-E & {\left[\int_{G} \hat{p}(T, x) \cdot \tilde{Y}(T, x) d x\right] } \\
=-E & {\left[\int _ { G } \left(\hat{p}(0, x) \cdot \tilde{Y}(0, x)+\int_{0}^{T}\{\tilde{Y}(t, x) d \hat{p}(t, x)+\hat{p}(t, x) d \tilde{Y}(t, x)\right.\right.} \\
& +(\sigma-\hat{\sigma}) \cdot \hat{q}(t, x)\} d t) d x] \\
=-E & {\left[\int _ { G } \left(\int _ { 0 } ^ { T } \left\{\tilde{Y}(t, x)\left[-\left(\frac{\partial H}{\partial y}\right)^{\wedge}-L^{*} \hat{p}(t, x)\right]\right.\right.\right.} \\
& +\hat{p}(t, x)[L \tilde{Y}(t, x)+(b-\hat{b})]+(\sigma-\hat{\sigma}) \hat{q}(t, x)\} d t) d x],
\end{aligned}
$$

where

$$
\left(\frac{\partial H}{\partial y}\right)^{\wedge}=\frac{\partial H}{\partial y}(t, x, \widehat{Y}(t, x), \hat{u}(t, x), \hat{p}(t, x), \hat{q}(t, x)) .
$$

Combining (2.11) and (2.15) we get

$$
\begin{gathered}
J(\hat{u})-J(u)=I_{1}+I_{2} \geq E\left[\int_{0}^{T}\left(\int_{G}\left\{\tilde{Y} L^{*} \hat{p}-\hat{p} \cdot L \tilde{Y}\right\} d x\right) d t\right] \\
+E\left[\int_{G}\left(\int_{0}^{T}\left\{\widehat{H}-H+\left(\frac{\partial H}{\partial y}\right)^{\wedge} \cdot \tilde{Y}(t, x)\right\} d t\right) d x\right]
\end{gathered}
$$

By the first Green formula (see e.g. [W, (20), page 258]) there exist first order boundary differential operators $A_{1}, A_{2}$ such that

$$
\int_{G}\left\{\widetilde{Y} L^{*} \hat{p}-\hat{p} L \widetilde{Y}\right\} d x=\int_{\partial G}\left\{\widetilde{Y} A_{1} \hat{p}-\hat{p} A_{2} \widetilde{Y}\right\} d S,
$$

where the integral on the right is the surface integral over $\partial G$.

By (1.3) and (2.5) we have $\widetilde{Y}(t, x)=\hat{p}(t, x)=0$ for all $(t, x) \in(0, T) \times \partial G$. Hence

$$
\int_{G}\left\{\tilde{Y} L^{*} \hat{p}-\hat{p} \cdot L \tilde{Y}\right\} d x=0 \quad \text { for all } \quad t \in(0, T) .
$$

Therefore (2.16) gives

$$
J(\hat{u})-J(u) \geq E\left[\int_{G}\left(\int_{0}^{T}\left\{\widehat{H}-H+\left(\frac{\partial H}{\partial y}\right)^{\wedge} \cdot \widetilde{Y}(t, x)\right\} d t\right) d x\right] .
$$


Since $H(y, u)$ is concave (by $(2.6))$, we have

$$
H-\widehat{H} \leq \frac{\partial H}{\partial y}(\widehat{Y}, \hat{u}) \cdot(Y-\widehat{Y})+\frac{\partial H}{\partial u}(\widehat{Y}, \hat{u})(u-\hat{u})
$$

Since $v \rightarrow H(\widehat{Y}, v)$ is maximal at $v=\hat{u}$ by $(2.7)$, we have

$$
\frac{\partial H}{\partial u}(\widehat{Y}, \hat{u}) \cdot(u-\hat{u}) \leq 0 \text {. }
$$

Hence by $(2.20)$

$$
H-\widehat{H}-\frac{\partial H}{\partial y}(\widehat{Y}, \hat{u}) \cdot(Y-\widehat{Y}) \leq 0
$$

which by (2.19) gives that

$$
J(\hat{u})-J(u) \geq 0 .
$$

Since $u \in \mathcal{A}$ was arbitrary the proof is complete.

In some applications the Hamiltonian function

$$
h(t, x, y, u):=H(t, x, y, u, \hat{p}(t, x), \hat{q}(t, x))
$$

is not concave in both variables $(y, u)$. In such cases it is useful to replace the concavity in $(y, u)$ by a weaker condition, sometimes called the Arrow condition:

(2.24) The function $\hat{h}(t, x, y):=\max _{v \in U} h(t, x, y, v)$ exists and is concave in $y$, for all $t, x$.

Then we get the following result:

\section{Theorem 2.2 (Sufficient SPDE maximum principle II)}

Let $\hat{u}, \widehat{Y}, \hat{p}, \hat{q}$ be as in Theorem 2.1. Suppose that $g(x, y)$ is concave in $y$ and that the maximum condition (2.7) and the Arrow condition (2.24) hold. Then $\hat{u}(t, x)$ is an optimal control for the stochastic control problem (1.6).

Proof. We proceed as in the proof of Theorem 2.1 up to and including (2.19). Then, to obtain (2.22) note that

$$
\begin{aligned}
H- & \widehat{H}-\frac{\partial H}{\partial y}(\widehat{Y}, \hat{u}) \cdot(Y-\widehat{Y}) \\
& =h(t, x, Y(t, x), u(t, x))-h(t, x, \widehat{Y}(t, x), \hat{u}(t, x)) \\
& -\frac{\partial h}{\partial y}(t, x, \widehat{Y}(t, x), \hat{u}(t, x)) \cdot(Y(t, x)-\widehat{Y}(t, x))
\end{aligned}
$$

This is $\leq 0$ by the same argument as in the proof of the Arrow sufficiency theorem for the deterministic case. See [SS, Theorem 5, p. 107-108]. For completeness we give the details:

Note that by (2.7) we have

$$
h(t, x, \widehat{Y}(t, x), \hat{u}(t, x))=\hat{h}(t, x, \widehat{Y}(t, x)) .
$$


Moreover, by definition of $\hat{h}$ in $(2.24)$ we have

$$
h(t, x, y, u) \leq \hat{h}(t, x, y) \quad \text { for all } t, x, y, u .
$$

Therefore, subtracting (2.25) from (2.26) we get

$$
\begin{array}{r}
h(t, x, y, u)-h(t, x, \widehat{Y}(t, x), \hat{u}(t, x)) \\
\leq \hat{h}(t, x, y)-\hat{h}(t, x, \widehat{Y}(t, x)) \quad \text { for all } t, x, y, u .
\end{array}
$$

Hence, to prove (2.22) it suffices to prove that

$$
\begin{aligned}
& \hat{h}(t, x, Y(t, x))-\hat{h}(t, x, \widehat{Y}(t, x)) \\
& \quad-\frac{\partial h}{\partial y}(t, x, \widehat{Y}(t, x), \hat{u}(t, x)) \cdot(Y(t, x)-\widehat{Y}(t, x)) \leq 0 \quad \text { for all } t, x .
\end{aligned}
$$

Fix $(t, x) \in[0, T] \times \bar{G}$.

By concavity of the function $y \rightarrow \hat{h}(t, x, y)$ it follows by a standard separating hyperplane argument (see e.g. [R, Chapter 5 , Section 23]) that there exists a supergradient $a \in \mathbb{R}$ for $\hat{h}(t, x, y)$ at $y=\widehat{Y}(t, x)$, i.e.

$$
\hat{h}(t, x, y)-\hat{h}(t, x, \widehat{Y}(t, x))-a \cdot(y-\widehat{Y}(t, x)) \leq 0 \quad \text { for all } y .
$$

Define

$$
\phi(y)=h(t, x, y, \hat{u}(t, x))-h(t, x, \widehat{Y}(t, x), \hat{u}(t, x))-a \cdot(y-\widehat{Y}(t, x)) ; \quad y \in \mathbb{R} .
$$

Then by (2.27) and (2.29) we have

$$
\phi(y) \leq 0 \quad \text { for all } y \in \mathbb{R} .
$$

Moreover, we clearly have

$$
\phi(\widehat{Y}(t, x))=0
$$

Therefore

$$
\phi^{\prime}(\widehat{Y}(t))=\frac{\partial h}{\partial y}(t, x, \widehat{Y}(t, x), \hat{u}(t, x))=a .
$$

Combining this with (2.29) we obtain (2.28) and the proof is complete.

\section{Controls which do not depend on $x$}

In some cases, for example in the application to partial observation control (see e.g. [B1], [B2], [B3], [P1], [P2]), it is of interest to consider only controls $u(t)=u(t, \omega)$ which do not depend on the space variable $x$. Let us denote the set of such controls $u \in \mathcal{A}$ by $\mathcal{A}_{1}$. Then the problem corresponding to (1.6) is to find $J_{1}^{*} \in \mathbb{R}$ and $u^{*} \in \mathcal{A}_{1}$ such that

$$
J_{1}^{*}=\sup _{u \in \mathcal{A}_{1}} J(u)=J\left(u^{*}\right)
$$

where

$$
J(u)=E\left[\int_{0}^{T}\left(\int_{G} f(t, x, Y(t, x), u(t)) d x\right) d t+\int_{G} g(x, Y(T, x)) d x\right]
$$

and $Y(t, x)$ is as before given by (1.1)-(1.3) (but with $u(t, x)$ replaced by $u(t)$ ).

To handle this situation, we modify Theorem 2.1 as follows: 


\section{Theorem 2.3 (Sufficient SPDE maximum principle III)}

Let $\hat{u}=\hat{u}(t) \in \mathcal{A}_{1}$ with corresponding solution $\widehat{Y}(t, x)$ of $(1.1)-(1.3)$ and let $\hat{p}(t, x), \hat{q}(t, x)$ be a solution of the associated adjoint backward SPDE (2.3)-(2.5). Assume that (2.6) and (2.30) hold, where

$$
\begin{aligned}
& \text { (Average maximum condition) } \\
& \qquad \begin{array}{l}
\int_{G} H(t, x, \widehat{Y}(t, x), \hat{u}(t), \hat{p}(t, x), \hat{q}(t, x)) d x \\
\quad=\sup _{u \in U}\left\{\int_{G} H(t, x, \widehat{Y}(t, x), u, \hat{p}(t, x), \hat{q}(t, x)) d x\right\}
\end{array}
\end{aligned}
$$

Then $\hat{u}(t)$ is an optimal control for the problem (2.28)-(2.29).

Proof of Theorem 2.3. We proceed as in the proof of Theorem 2.1: Let $u \in \mathcal{A}_{1}$ with corresponding solution $Y(t, x)$ of (1.1)-(1.3). Consider

$$
J(\hat{u})-J(u)=E\left[\int_{0}^{T} \int_{G}\{\hat{f}-f\} d x d t+\int_{G}\{\hat{g}-g\} d x\right]
$$

where

$$
\begin{aligned}
& \hat{f}=f(t, x, \widehat{Y}(t, x), \hat{u}(t)), \quad f=f(t, x, Y(t, x), u(t)), \\
& \hat{g}=g(x, \widehat{Y}(T, x)), \quad \text { and } \quad g=g(x, Y(T, x)) \text {. }
\end{aligned}
$$

Using a similar shorthand notation for $b=b(t, x, Y(t, x), u(t)), \hat{b}, \sigma$ and $\hat{\sigma}$ and setting

$$
\begin{aligned}
\widehat{H} & =H(t, x, \widehat{Y}(t, x), \hat{u}(t,), \hat{p}(t, x), \hat{q}(t, x)), \\
H & =H(t, x, Y(t, x), u(t), p(t, x), q(t, x))
\end{aligned}
$$

we see that (2.31) can be written

$$
J(\hat{u})-J(u)=I_{1}+I_{2}
$$

where

$$
I_{1}=E\left[\int_{0}^{T} \int_{G}\{\widehat{H}-H-(\hat{b}-b) \hat{p}-(\hat{\sigma}-\sigma) \hat{q}\} d x d t\right]
$$

and

$$
I_{2}=E\left[\int_{G}\{\hat{g}-g\} d x\right]
$$

By concavity of the function $y \rightarrow g(x, y)$ we have

$$
\int_{G}\{g(x, Y(T, x))-g(x, \widehat{Y}(T, x))\} d x \leq \int_{G} \frac{\partial g}{\partial y}(x, \widehat{Y}(T, x)) \cdot \widetilde{Y}(T, x) d x
$$

where

$$
\widetilde{Y}(t, x)=Y(t, x)-\widehat{Y}(t, x) .
$$


Therefore we get, as in the proof of Theorem 2.1,

$$
\begin{aligned}
I_{2} \geq-E & {\left[\int _ { 0 } ^ { T } \left(\int _ { G } \left\{\widetilde{Y}(t, x)\left[-\left(\frac{\partial H}{\partial y}\right)^{\wedge}-L^{*} \hat{p}(t, x)\right]\right.\right.\right.} \\
& +\hat{p}(t, x)[L \widetilde{Y}(t, x)+(b-\hat{b})]+(\sigma-\hat{\sigma}) \hat{q}(t, x)\} d x) d t]
\end{aligned}
$$

where

$$
\left(\frac{\partial H}{\partial y}\right)^{\wedge}=\frac{\partial H}{\partial y}(t, x, \widehat{Y}(t, x), \hat{u}(t), \hat{p}(t, x), \hat{q}(t, x)) .
$$

Summing (2.35) and (2.38) we get, as in (2.17),

$$
J(\hat{u})-J(u)=I_{1}+I_{2} \geq E\left[\int_{0}^{T}\left(\int_{G}\left\{\widehat{H}-H+\tilde{Y} \cdot\left(\frac{\partial H}{\partial y}\right)^{\wedge}\right\} d x\right) d t\right] .
$$

where $\widehat{H}$ and $H$ are given (3.32) and (2.33). Since $(y, u) \rightarrow H(y, u)$ is concave (by (2.6)), we have

$$
H-\widehat{H} \leq \frac{\partial H}{\partial y}(\widehat{Y}, \hat{u}) \cdot(Y-\widehat{Y})+\frac{\partial H}{\partial u}(\widehat{Y}, \hat{u}) \cdot(u-\hat{u})
$$

Combining (2.39) and (2.40) we get

$$
\begin{aligned}
J(\hat{u})-J(u) \geq E\left[\int_{0}^{T}\left(\int_{G}-\frac{\partial H}{\partial u}(\widehat{Y}, \hat{u}) \cdot(u-\hat{u}) d x\right) d t\right] \\
=-E\left[\int_{0}^{T}(u-\hat{u}) \cdot \frac{\partial}{\partial u}\left(\int_{G} H(t, x, \widehat{Y}, u, \hat{p}, \hat{q}) d x\right)_{u=\hat{u}(t)} d t\right] \geq 0, \\
\text { since } u=\hat{u}(t) \text { maximizes } u \rightarrow \int_{G} H(t, x, \widehat{Y}, u, \hat{p}, \hat{q}) d x
\end{aligned}
$$

by assumption (2.30).

\section{Applications}

We now illustrate the results of Section 2 by looking at some examples.

\section{Example 3.1 (Optimal harvesting I)}

Suppose the density $Y(t, x)$ of a population (e.g. fish) at time $t \in(0, T)$ and at the point $x \in G \subset \mathbb{R}^{n}$ is given by the stochastic reaction-diffusion equation

$$
\begin{aligned}
& d Y(t, x)=\left[\frac{1}{2} \Delta Y(t, x)+\alpha Y(t, x)-u(t, x)\right] d t+\beta Y(t, x) d B(t) \\
& \text { (where } \Delta=\sum_{i=1}^{n} \frac{\partial^{2}}{\partial x_{i}^{2}} \text { is the Laplacian) }
\end{aligned}
$$

with boundary conditions

$$
\begin{array}{llrl}
Y(0, x)=\xi(x) ; & & x \in \bar{G} \\
Y(t, x)=\eta(t, x) ; & & (t, x) \in(0, T) \times \partial G .
\end{array}
$$


Here $u(t, x) \geq 0$ is our harvesting rate at $(t, x)$.

See e.g. [S] for more information on reaction-diffusion equations. A special class of stochastic reaction-diffusion equations is studied in [ØVZ1] and [ØVZ2].

Suppose we want to maximize a combination of the total expected utility of the consumption and the terminal size of the population, expressed by the performance criterion

$$
J(u)=E\left[\int_{0}^{T}\left(\int_{G} \frac{u^{\gamma}(t, x)}{\gamma} d x\right) d t+\theta \int_{G} Y(T, x) d x\right]
$$

where $\gamma \in(0,1)$ and $\theta>0$ are given constants. In this case the Hamiltonian (2.1) gets the form

$$
H(t, x, y, u, p, q)=\frac{u^{\gamma}}{\gamma}+(\alpha y-u) p+\beta y q
$$

Therefore the adjoint equations (2.3)-(2.5) become

$$
\begin{aligned}
& \begin{array}{l}
d p(t, x)=-\left[\alpha p(t, x)+\beta q(t, x)+\frac{1}{2} \Delta p(t, x)\right] d t \\
+q(t, x) d B(t) ; \quad(t, x) \in(0, T) \times G
\end{array} \\
& \begin{array}{l}
p(T, x)=\theta ; \quad x \in G \\
p(t, x)=0 ; \quad(t, x) \in(0, T) \times \partial G .
\end{array}
\end{aligned}
$$

Because the boundary conditions and all the coefficients are deterministic, we see that we can choose $q(t, x)=0$ and solve (3.6)-(3.8) for deterministic $p(t, x)$. The equation (3.6) then gets the form

$$
\frac{\partial p}{\partial t}(t, x)+\frac{1}{2} \Delta p(t, x)+\alpha p(t, x)=0 ; \quad(t, x) \in(0, T) \times G .
$$

It is well-known that the boundary value problem (3.7)-(3.9) has the unique solution

$$
p(t, x)=\theta e^{\alpha T} P\left[W^{x}(s) \in G \text { for all } s \in[t, T]\right],
$$

where $W^{x}(\cdot)$ denotes $n$-dimensional Brownian motion starting at $x \in \mathbb{R}^{n}$ with probability law P. (See e.g. [KS, Chapter 4] or [Ø, Chapter 9].)

The function

$$
u \rightarrow H(t, x, y, u, p, q)=\frac{u^{\gamma}}{\gamma}+(\alpha y-v) p+\beta y q ; \quad u \geq 0
$$

is maximal when

$$
u=\hat{u}(t, x)=(p(t, x))^{\frac{1}{\gamma-1}}
$$

where $p(t, x)$ is given by $(3.10)$.

With this choice of $\hat{u}(t, x)$ we see that all the conditions of Theorem 2.1 are satisfied and we conclude that $\hat{u}(t, x)$ is an optimal harvesting rate.

\section{Example 3.2 (Optimal harvesting II)}

Supppose we modify the performance criterion $J(u)$ of Example 3.1 to

$$
J_{0}(u)=E\left[\int_{0}^{T}\left(\int_{\mathbb{R}} \frac{u^{\gamma}(t, x)}{\gamma} d x\right) d t+\int_{\mathbb{R}} g(x, Y(T, x)) d x\right]
$$


where $g: \mathbb{R} \rightarrow \mathbb{R}$ is a given $C^{1}$-function. The Hamiltonian $H(t, x, y, p, q)$ remains the same and so the candidate $\widehat{u}(t, x)$ for the optimal control has the same form as in (3.11), i.e.

$$
\widehat{u}(t, x)=(p(t, x))^{\frac{1}{\gamma-1}}
$$

The difference is that now we have to work harder to find $p(t, x)$. The backward stochastic partial differential equation for $p(t, x)$ is now

$$
\begin{aligned}
& d p(t, x)=-\left[\alpha p(t, x)+\beta q(t, x)+\frac{1}{2} \Delta p(t, x)\right] d t+q(t, x) d B(t) ; \quad(t, x) \in(0, T) \times \mathbb{R} \\
& p(T, x)=F(x, \omega) ; \quad x \in \mathbb{R} \\
& \lim _{|x| \rightarrow \infty} p(t, x)=0 ; \quad t \in(0, T)
\end{aligned}
$$

where we have put

$$
F(x, \omega)=\frac{\partial g}{\partial y}(x, Y(T, x)) ; \quad x \in \bar{G} .
$$

To solve this equation we proceed as follows:

First note that if we put

$$
\widetilde{p}(t, x):=e^{\alpha t} p(t, x)
$$

then (3.14)-(3.16) get the form

$$
\begin{aligned}
& d \widetilde{p}(t, x)=-\beta e^{\alpha t} q(t, x) d t-\frac{1}{2} \Delta \widetilde{p}(t, x) d t+e^{\alpha t} q(t, x) d B(t) ; \quad(t, x) \in(0, T) \times \mathbb{R} \\
& \widetilde{p}(T, x)=e^{\alpha T} F(x, \omega) ; \quad x \in \mathbb{R} \\
& \lim _{|x| \rightarrow \infty} \widetilde{p}(t, x)=0 ; \quad t \in(0, T) .
\end{aligned}
$$

Next, define the measure $P_{0}$ by

$$
d P_{0}(\omega)=\exp \left(\beta B(t)-\frac{1}{2} \beta^{2} t\right) d P(\omega) \quad \text { on } \quad \mathcal{F}_{T} .
$$

Then by the Girsanov theorem the process

$$
B_{0}(t):=-\beta t+B(t) ; \quad 0 \leq t \leq T
$$

is a Brownian motion w.r.t. $P_{0}$.

Suppose $F(x, \cdot) \in L^{2}\left(P_{0}\right)$ for each $x$. Then by the Itô representation theorem there exists a unique adapted process $\psi(t, x, \omega)$ such that $E_{0}\left[\int_{0}^{T} \psi^{2}(t, x, \omega) d t\right]<\infty$ and

$$
e^{\alpha T} F(x, \omega)=h(x)+\int_{0}^{T} \psi(t, x, \omega) d B_{0}(t),
$$

where $h(x)=E_{0}\left[e^{\alpha T} F(t, \cdot)\right]$ and $E_{0}$ denotes expectation w.r.t. $P_{0}$. 
Define the heat operator $Q_{t}$ by

$$
\left(Q_{t} f\right)(x)=(2 \pi t)^{-1 / 2} \int_{\mathbb{R}} f(y) \exp \left(-\frac{|x-y|^{2}}{2 t}\right) d y ; \quad f \in \mathcal{D},
$$

where $\mathcal{D}$ is the set of real functions on $\mathbb{R}$ for which the integral converges. Now define

$$
\begin{aligned}
\widetilde{p}(t, x): & =Q_{T-t}\left(\int_{0}^{t} \psi(s, \cdot, \omega) d B_{0}(s)+h(\cdot)\right)(x) \\
& =\int_{0}^{T}\left(Q_{T-t} \psi(s, \cdot, \omega)\right)(x) d B_{0}(s)+\left(Q_{T-t} h\right)(x) .
\end{aligned}
$$

Then, by well-known properties of the $Q_{t}$ operator,

$$
\begin{aligned}
d \widetilde{p}(t, x)= & {\left[\int_{0}^{T}+\frac{1}{2} \Delta\left(Q_{T-t} \psi(s, \cdot, \omega)\right)(x) d B_{0}(s)-\frac{1}{2} \Delta\left(Q_{T-t} h\right)(x)\right] d t } \\
& +\left(Q_{T-t} \psi(t, \cdot, \omega)\right)(x) d B_{0}(t) \\
= & -\frac{1}{2} \Delta \widetilde{p}(t, x) d t+q(t, x) d B_{0}(t),
\end{aligned}
$$

where

$$
q(t, x)=\left(Q_{T-t} \psi(t, \cdot, \omega)\right)(x) .
$$

By (3.22) we see that (3.26) is identical to (3.19). We have proved

Theorem 3.3 Suppose

$$
\int_{\mathbb{R}}\left(E_{0}\left[F^{2}(y, \cdot)\right]\right)^{1 / 2} \exp \left(-\frac{y^{2}}{2}\right) d y<\infty .
$$

Then the solution $(p(t, x), q(t, x))$ of the backward SPDE (3.14)-(3.16) is given by

$$
p(t, x)=e^{-\alpha t} \widetilde{p}(t, x) \quad \text { with } \quad \widetilde{p}(t, x) \text { as in (3.25) }
$$

and

$$
q(t, x)=\left(Q_{T-t} \psi(t, \cdot, \omega)\right)(x),
$$

with $\psi$ given implicitly by (3.23).

For general existence and uniqueness results for backward stochastic partial differential equations see $[\varnothing \mathrm{Z}]$.

Acknowledgments I am grateful to Mark Davis, David Lefèvre, Agnès Sulem and Tusheng Zhang for helpful comments. 


\section{References}

[B1] A. Bensoussan: Maximum principle and dynamic programming approaches of the optimal control of partially observed diffusions. Stochastics 9 (1983), 169-222.

[B2] A. Bensoussan: Stochastic maximum principle for systems with partial information and application to the separation principle. In M. Davis and R. Elliott (editors): Applied Stochastic Analysis. Gordon \& Breach 1991, pp. 157-172.

[B3] A. Bensoussan: Stochastic Control of Partially Observable Systems. Cambridge University Press 1992.

[KS] I. Karatzas and S.E. Shreve: Brownian Motion and Stochastic Calculus. Second Edition. Springer-Verlag 1991.

[M] R. E. Mortensen: Stochastic optimal control with noisy observations. Int. J. Control 4 (1966), 455-464.

[Ø] B. Øksendal: Stochastic Differential Equations. Sixth Edition. Springer-Verlag 2003.

[ØVZ1] B. Øksendal, G. Våge and H. Zhao: Asymptotic properties of the solutions to stochastic KPP equations. Proc. Royal Soc. Edinburgh 130A (2000), 1363-1381.

[ØVZ2] B. Øksendal, G. Våge and H. Zhao: Two properties of stochastic KPP equations: Ergodicity and pathwise property. Nonlinearity 14 (2001), 639-662.

[ØZ] B. Øksendal and T. Zhang: On backward stochastic partial differential equations. Preprint, Dept. of Mathematics, University of Oslo 18/2001.

[P1] E. Pardoux: Stochastic partial differential equations and filtering of diffusion processes. Stochastics 3 (1979), 127-167.

[P2] E. Pardoux: Filtrage non lineaire et équations aux derivées partielles stochastiques associées. Ecole d'Été de Probabilités de Saint-Flour 1989.

[R] R. T. Rockafellar: Convex Analysis. Princeton University Press 1970.

[S] J. Smoller: Shock Waves and Reaction-Diffusion Equations. Springer-Verlag 1983.

[SS] A. Seierstad and K. Sydsæter: Optimal Control Theory with Economic Applications. North-Holland 1987.

[W] J. Wloka: Partial Differential Equations. Cambridge Univ. Press 1987. 